\title{
Colour changes as an indicator for estimating fungal growth in solid state fermentation
}

\author{
Musaalbakri Abdul Manan ${ }^{1,2}$ and Colin Webb ${ }^{2}$ \\ 1Enzyme and Fermentation Technology Programme, Biotechnology and Nanotechnology Research Centre, Malaysian \\ Agricultural Research and Development Institute, Persiaran MARDI - UPM, 43400 Serdang, Selangor Malaysia. \\ 2Satake Centre for Grain Process Engineering, School of Chemical Engineering and Analytical Science, Faculty of \\ Engineering and Physical Sciences, University of Manchester, United Kingdom. \\ Email: colin.webb@machester.ac.uk
}

Received 31 October 2016 ; Received in revised form 5 June 2017; Accepted 20 June 2017

\begin{abstract}
Aims: In this study, measurement of colour changes during solid state fermentation (SSF) are presented as one of the potential techniques that can be used to describe growth, complementary to other biomass estimation, such as weight of fungus, spores concentration, organic matter loss, glucosamine and enzyme activity, which is directly related to growth.

Methodology and results: In this study, fungal fermentation of Aspergillus awamori and A. oryzae are carried out on complex heterogeneous solid media; wheat bran, soybean hull and rapeseed meal, which are constituted of various soluble and insoluble solid particles. Fermented mass was extracted using distilled water to obtain a cell free extract, which can be determined quickly and accurately using UV-Visible spectrophotometer measured absorbance at $300 \mathrm{~nm}$. The results showed a significant correlation between colours produced from fungal SSF and the concentration of spores, weight of fungus cells, organic matter loss, glucosamine and enzyme activity. We found that the colour density proportionally increased when the studied parameters mentioned above increased. For the growth of $A$. awamori and $A$. oryzae on wheat bran, soybean hulls and rapeseed meal, it was confirmed that colour production was directly proportional to fungal growth. In general, colour-based methods seem to be the most promising approach for biomass estimation in SSF.

Conclusions, significance and impacts of study: The theory of measuring colour changes in SSF by UV-visible spectroscopy demonstrates that the colour method gives some valuable information than just obtaining a visual observation or spore count to describe growth of fungal mycelium in SSF. The ideas obtained from this exercise might provide a quick and convenient method for quality control of fungal growth. The advantages of this method are that the procedure is simple to carry out, it is non-destructive and no special and expensive reagents are required and the process is very cheap. This new approach is an important complimentation to the existing techniques especially for basic studies.
\end{abstract}

Keywords: peat swamp forest, soil bacteria, peat water, lignocellulose

\section{INTRODUCTION}

Solid state fermentation (SSF) can be defined as process that involves the growth of microorganisms on moist particles of solid materials in beds in which the spaces between the particles are filled with a continuous gas phase (Mitchell et al., 2010; 2011;). In contrast to submerged fermentation (SmF), SSF does not allow accurate biomass estimation. However, SmF faces the same problem when the fermentation media contain solid particles. It is difficult to estimate biomass in SSF directly because it is very difficult to separate the microorganisms and the solid particles. This is more the case for fermentations involving filamentous fungi because fungal mycelium penetrates deeply into the substrate and becomes inextricably entangled within the solids. Chattaway et al. (1992) stated "in the case of biomass estimation, elemental balances are useful either for obtaining on-line estimates or for calculating biomass offline, when direct measurement is difficult, during fermentations on solid-containing media". Although there are a large number of studies on biomass estimation in SSF, the available methods do not perform well. There are operational and sample preparation difficulties around these existing methods. For example, dry weight measurements (including both cells and the solid particles), are used to estimate cell biomass concentration, resulting inaccurate data. However, Hashemi et al. (2011) investigated a feasible approach for modelling the phases of the bacterial growth curve and production of $\alpha$-amylase by Bacillus sp. in a SSF process based on variation in dry weight. Ramana Murthy et al. (1993) monitored biomass in SSF using light reflectance. They observed colour changes due to the growth of the 
fungus during SSF. They used CIE (Commission International de l'Eclairage) system based on the principles of additive colour mixing. The authors concluded the correlation between the results of the proposed technique and the glucosamine concentration data was showing that a direct and linear relationship.

Achieving an accurate result in determining biomass remains a major challenge in SSF. According to Borzani, (2000) measuring growth through a direct or an indirect technique is important to provide a better understanding of process control and also to give an idea regarding the productivity of a cell culture. Fungi are well-characterised microorganisms and are widely used in SSF due to their ability to colonise and penetrate into the solid substrate. The compressed structure of the mycelia and the solid substrate does not allow a complete recovery of the biomass, which may not be insurmountable. Although significant advances have been made with the availability of various techniques; however, progress has been very unsatisfactory. The evaluation of microbial growth in SSF may sometimes become laborious, impractical and inaccurate (Wei et al., 2006). Essentially, this remains another critical issue for monitoring growth. Since the use of a direct technique such as the dry weight method is impractical, the use of an indirect estimation technique is the only alternative. A range of indirect methods can be used to estimate biomass, which can be classified into six categories: (1) Measuring cell components not present in the substrate (eg. glucosamine); (2) measuring biomass component present in both substrate and biomass (eg. protein); (3) measuring other secondary metabolites (eg. penicillin); (4) measuring metabolic activity (eg. oxygen consumed, carbon dioxide produce and metabolic heat); (5) measuring images from direct microscopic observation (eg. scanning electron microscope or confocal microscopy) and (6) measuring biomass from the substrate matrix (Abdul Manan and Webb, 2018a). According to Mitchell et al. (2004; 2006), oxygen uptake rate (OUR) and and carbon dioxide evolution rate (CER) offer the advantage of a fast response time and are directly linked to the metabolism of the microorganism. This is also true for the heat evolved during fermentation as a result of microbial metabolism (Harris and Kell, 1985). Kabanova et al. (2012) developed a method for the investigation of bacterial growth, growth of colonies in solid-state matrices using isothermal microcalorimetry. They showed that microcalorimetry measurements was a very powerful instrument in studying quantitative detailed peculiarities of SSF and it is possible to calculate heats evolved during the growth with numbers of bacteria in the sample. The information of the profile of fungal biomass growth throughout any SSF process constitutes an essential parameter in estimation of kinetic variables and subsequently, scale-up of the process (Koutinas et al., 2003).

In order for a particular strategy to be useful, it is necessary to have analytical methods for the analysis and evaluation of fungal growth. Such quantification is possible by measuring the colour produced in SSF using colourimetric technique. As an extension of previous report (Abdul Manan, 2016; Abdul Manan and Webb, $2018 \mathrm{~b}$ ), in the present work, experiments were carried out to quantify the colour of fermentation medium as a means for estimating growth. It is a general observation that the colour or pigment of the fermenting solid substrate changes during the course of the SSF due to the growth of the fungus. During the course of the investigation of fungal growth on solid substrate, it was found that the colour or pigment of the solid substrate became darker with progressive growth. The main aim of this study is to provide better insight into monitoring biomass growth in $A$. awamori and $A$. oryzae SSF using colourimetric technique. Other objective of this study is to investigate the correlation between colourimetric measurement and several available biomass estimation techniques such as weight of fungus, spores concentration, organic matter loss, glucosamine and enzyme activity.

\section{MATERIALS AND METHODS}

\section{Microorganisms}

Aspergillus awamori and Aspergillus oryzae obtained from the School of Chemical Engineering and Analytical Science, Faculty of Engineering and Physical Science, University of Manchester are used throughout this study. Fungal spores in universal bottle were stored at $4{ }^{\circ} \mathrm{C}$ in agar slopes of solid sporulation medium containing $5 \%$ (w/v) whole wheat flour and $2 \%(w / v)$ agar (SigmaAldrich) as a stock culture and they were sub-cultured in the time interval of every two months. This strain produces compact colonies with huge spores and accumulates large quantities of black and green pigments during growth in the form of mycelium for $A$. awamori and A. oryzae, respectively (Figure 1 ).
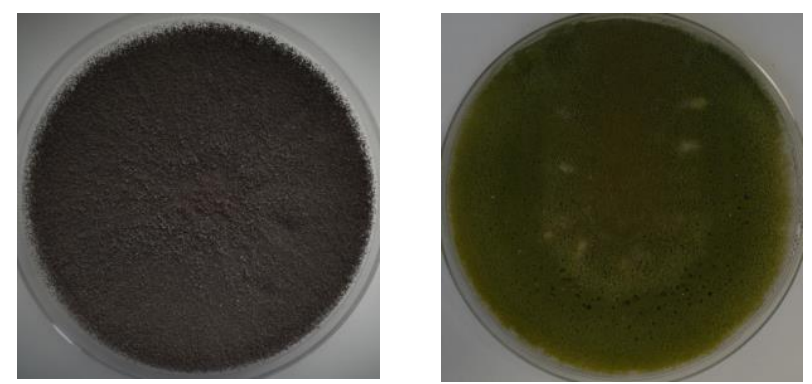

Figure 1: $A$. awamori (left) and $A$. oryzae (right) on whole wheat flour agar plate. Growth of colonies after 7 days of incubation at $30^{\circ} \mathrm{C}$.

\section{Inoculum preparation}

Fungal progeny obtained with monospore isolation of $A$. awamori and $A$. oryzae, were used throughout this study. For preparing a spore suspension, well-sporulated slants of both fungi were used. The spores were washed by lightly scrapping the spores with a wire loop and dipped in $10.0 \mathrm{~mL}$ of sterile $0.1 \%(\mathrm{v} / \mathrm{v})$ Tween 80 . The spore suspension $(0.5 \mathrm{~mL})$ was further transferred onto the surface of $100.0 \mathrm{~mL}$ of sporulation medium in a $500.0 \mathrm{~mL}$ Erlenmeyer flask and incubated for 7 days at $30^{\circ} \mathrm{C}$. After the incubation period, $50.0 \mathrm{~mL}$ of sterile $0.1 \%(\mathrm{v} / \mathrm{v})$ Tween 80 solution and several sterile glass beads (4-mm diameter) were added to the flask. The spores were suspended by shaking the flask gently and collected in one bottle as a spore suspension. The concentration of 
the spore suspension was measured by haemocytometer. The volume of suspension needed for the inoculation of the solid substrate was calculated for each experiment to reach a concentration of around $1.0 \times 10^{6} \mathrm{spores} / \mathrm{g}$ solid substrate. Fungal spores in universal bottles were stored at $4{ }^{\circ} \mathrm{C}$ in agar slopes of solid sporulation medium, containing $5 \%(\mathrm{w} / \mathrm{v})$ whole wheat flour and $2 \%(\mathrm{w} / \mathrm{v})$ agar as a stock culture.

\section{Fermentation media and preparation}

\section{Solid state fermentation}

All three selected substrates (wheat bran, soybean hulls and rapeseed meal) were used without any treatment as a solid medium for growing $A$. awamori and $A$. oryzae. Wheat bran was obtained from Cargill Wheat Processing Plant, Manchester, UK. Soybean hulls and rapeseed meal were obtained from Brocklebank Oilseed Processing Division, Cargill Wheat Processing Plant, Liverpool, UK. All experiments were carried out in $9.0 \mathrm{~cm}$ Petri dishes. $12.0 \mathrm{~g}$ of wheat bran and soybean hulls, and $15.0 \mathrm{~g}$ of rapeseed meal were used. These amounts were used to bring the height of the substrate bed in the Petri dishes to $1.0 \mathrm{~cm}$. After autoclaving, inoculum transfer was carried out aseptically, and sterile distilled water was used to moisten the solid substrates. The initial moisture content of the substrate was $65 \%$. After the inoculation process, all the Petri dishes were placed in a growth incubator with a strictly controlled temperature at $30^{\circ} \mathrm{C}$. At the end of the fermentation period (72 h), samples were taken out for colour analysis.

\section{Analytical method}

\section{Colour extraction}

During the fermentation process, a sample was taken at various time intervals for colour analysis. A normal observation in SSF is un-even growth on fermented substrate. This situation will usually result in unsatisfied outcome. Therefore, a standard procedure was developed in this study to obtain a satisfactory sampling process. To overcome this issue and to satisfy the need for a homogenous sampling process, an amount of fermented mass was harvested, crashed and vigorously mix using spatula. If the experiments were conducted in petri dishes, the whole fermented mass was blended using a foodprocessing blender.

\section{Samples from agar in Petri dish}

At the end of the incubation period, the colour was extracted from spores, mycelium and a combination the spores, mycelium and agar. Ten $\mathrm{mL}$ of sterile distilled water and several sterile glass beads (4 mm diameter) were added onto a petri dish. The spores were suspended by shaking the petri dish gently and collected in $250 \mathrm{~mL}$ flask as a spores suspension. This step was repeated until all the spores were harvested and the final volume of the spores suspension was made up to $40 \mathrm{~mL}$. The Petri dish with the washed spores from the previous step was used to obtain mycelial material. The surface was gently

scratched with a spatula to collect about $2.0 \mathrm{~g}$ (wet weight) of mycelium in $250 \mathrm{~mL}$ flask containing $40 \mathrm{~mL}$ distilled water.

One (1) piece of $4 \times 4 \mathrm{~mm}$ of mycelial blocks of $A$. awamori and $A$. oryzae were used to extract the colour in $250 \mathrm{~mL}$ flask containing $40 \mathrm{~mL}$ distilled water. All the flasks were kept shaking on an orbital rotary shaker (Infors AG-CH4103, Switzerland) at $30^{\circ} \mathrm{C}$ and $250 \mathrm{rpm}$ for $30 \mathrm{~min}$. The suspensions were then centrifuged (Eppendorf Centrifuge 5804, Germany) at 10,000 rpm for $10 \mathrm{~min}$ at $4{ }^{\circ} \mathrm{C}$. This procedure was repeated until a clear solution is obtained.

\section{Samples from SSF}

At the end of the incubation period, samples were harvested for analysis. Approximately $2.0 \mathrm{~g}$ (on a wet basis) from all the fermented samples was extracted with $40 \mathrm{~mL}$ distilled water and shaken for $1 \mathrm{~h}$ on a rotary shaker (Infors AG-CH4103, Switzerland) at $250 \mathrm{rpm}$ and $30{ }^{\circ} \mathrm{C}$. To make sure of obtaining very clean extraction solution free from spores, the suspensions were centrifuged using a laboratory centrifuge (Eppendorf Centrifuge 5804, Germany) at 10,000 rpm for 5 min before recovery by filtration through a filter paper (Whatman No. 1). Centrifugation was repeated when needed under similar conditions to achieve complete separation of spores or contaminating substances from the colour solution.

\section{Spectrophotometric analysis}

To identify the most suitable wavelength for absorbance measurement of the filtrate obtained using the extraction procedure, samples were scan over the wavelength range 200-800 $\mathrm{nm}$ and the wavelength corresponding to absorption maxima were determined. Water was used as a blank. Whenever necessary, the filtrate samples were diluted with distilled water prior to absorbance measurement. Colour production was calculated by multiplying the absorbance units by the dilution factor. The value was expressed as $\mathrm{UA}_{300} / \mathrm{g}$ of dried fermented substrate (dry basis) [db]. The readings of absorbance were used for the calculation of optical density using the formula below.

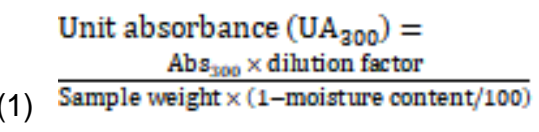

Colour yield was expressed as unit absorbance $\left(\mathrm{UA}_{300}\right)$ using its absorbance maximum per gram dry fermented substrate as described in Equation 1. It is important in this study that all the samples are analysed using a scanning spectrophotometer within the wavelength range of $200-800 \mathrm{~nm}$ to observe a typical normalised UV absorption spectrum. The spectrum of colour absorbance was obtained using a Shimadzu UVVis 2410PC series scanning spectrophotometer (Japan) taking into consideration the dilution factor of samples. 


\section{Biomass estimation}

\section{Spores concentration}

About $2.0 \mathrm{~g}$ (wet weight) of fermented substrate of $A$. awamori and $A$. oryzae were used to harvest the spores in a $250 \mathrm{~mL}$ flask containing $40 \mathrm{~mL} 0.1 \%(\mathrm{v} / \mathrm{v})$ Tween 80 . The flasks were kept shaking in orbital shaker at $30{ }^{\circ} \mathrm{C}$, agitated at $100 \mathrm{rpm}$ for $30 \mathrm{~min}$. Then the spore suspensions were filtered using a stainless steel sieve with aperture size $45 \mu \mathrm{m}$ to separate the solid particles. The concentration of the spore suspension was measured by haemocytometer. Spores suspension should be diluted enough so that the spores do not overlap with each other on the grid and should be uniformly distributed as it is assumed that the total volume in the chamber represents a random sample.

\section{Organic matter loss}

The technique for organic matter loss as described previously by Kumar et al. (2008) was followed. Biomass was estimated on the basis of loss of organic matter or dry solid during the course of fermentation. It was assumed that organic matter loss is due to carbon dioxide formation by fungal metabolic activities during the fermentation process. This technique involves a drying process at 80 ${ }^{\circ} \mathrm{C}$ using an oven.

\section{Glucosamine concentration}

The method was adapted from Scotti et al. (2001) and Swift (1973) with slight modification to suit the sample study. The method is based on the fact that glucosamine is a monomer component of chitin, present as acetylglucosamine. Chitin is an insoluble polymer present in the mycelium (Aidoo et al., 1982). The process consists of the polymerisation of chitin; followed by the liberated glucosamine. The glucosamine concentration throughout this study was expressed in units of milligrams per gram material measures on a dry basis (mg/g [db]).

\section{Protease activity}

During growth on solid substrates, filamentous fungi produce proteases in order to utilise the proteins found in the environment. Protease activity was evaluated by the formation of free amino nitrogen (FAN). FAN concentration was measured using the ninhydrin colourimetric method as outlined by the European Brewery Convention (Lie, 1973) with modifications made by Wang (1999). The method based on the colour reaction between ninhydrin and amino acids at $\mathrm{pH} 6.7$, which gives an estimate of amino acids, ammonia and in addition the terminal alpha-amino nitrogen groups of peptides and proteins. The amount of proteases for the production of one milligram FAN in one min under controlled conditions was defined as one unit of activity $(\mathrm{U} / \mathrm{mL})$. The protease activity throughout this study was expressed in units $\mathrm{U} / \mathrm{g}$ material measured on a dry basis.

\section{RESULTS AND DISCUSSION}

Based on the idea of estimation of the growth of the fungus Monascus purpureus, the analysis of the colour produced by micro-organisms is usually done by measuring the absorbance of colour solutions in the range near 400, 470 and $500 \mathrm{~nm}$ for yellow, orange and red pigments respectively (Wong and Koehler, 1981; Johns and Stuart, 1991; Lin et al., 1992; Juzlova et al., 1994).

In order to cover the objective of this work to determine whether the proposed hypothesis could be applied for quantifying fungal growth, different sets of experiments were conducted. Each one of the sets of experiments and fermentations were planned to address one or several of the objectives described above. Preliminary experiments in petri dishes were carried out in order to confirm the theory of using colour changes in fungal SSF as an approach for biomass estimation. All the parameters examined in these studies, which are the weight of fungi, spores concentration, organic matter loss, glucosamine concentration, and protease activity, are among the indirect techniques used for biomass estimation. The theory was tested by examining quantitatively the relationship between the above-mentioned parameters and colour changes that occur during fermentation.

The results obtained from these studies are described in the sections below. Typical normalised UV absorption spectra were obtained using a scanning spectrophotometer for each colour solution. These demonstrated that samples used in this work have very similar patterns. Multiple linear regressions were constructed in order to examine correlations between the concentration of colour produced and the indirect parameters studied. It is important to prove that the measurement of colour changes can provide a good correlation to growth of fungi in SSF.

The theory of measuring colour changes in SSF by UV-Vis spectroscopy demonstrates that this colourimetric technique provides valuable information complementary to visual observation and spore counting to describe the growth of fungal mycelium on SSF. Colour from cell free extracts absorbs light in the region $260-300 \mathrm{~nm}$ that largely corresponds to pigments in the supernatant. Colour density measured for fungal SSF in cell free extracts can be determined quickly and accurately using absorbance measurement at the chosen wavelength of $300 \mathrm{~nm}$. The advantages of this method include the fact that the procedure is cheap, fast, objective, simple to carry out, non-destructive and no special or expensive reagents are required. Making cell free extracts from fermented fungal biomass was carried only using distilled water and was performed in less than $2 \mathrm{~h}$. Water was efficient in the recovery of colour. Cell free extracts can also be used for multiple analyses including total reducing sugars, free amino nitrogen, glucose concentration, $\mathrm{pH}$ and enzymes activities. 


\section{Correlation between weight of fungus and colour density}

Growth and sporulation response was markedly affected by the solid medium used. Both fungi grew well on whole wheat flour agar as their sole source of carbon and energy. In both cases of $A$. awamori and $A$. oryzae, sporulation is highly efficient when fungi are grown on whole wheat flour agar and colour production was also the highest, followed by wheat bran agar, rapeseed meal agar and soybean hulls agar respectively. Figure 2 shows the relationship between colour density ( $\mathrm{UA}_{300 / \mathrm{g}}$ fungus) and weight of fungus ( $\mathrm{g} /$ Petri dish) for the period of 0 to 7 days. The colour density proportionally increased as weight/cell mass of fungus increased. The linear relationship of weight of fungus growing on different solid agar media with colour density was observed throughout the incubation period. Correlation by linear regression between fungal weight and $\mathrm{UA}_{300}$ for $A$. awamori and $A$. oryzae are represented in Figure 2. In all solid agars tested, correlation coefficients were greater than 0.98 and 0.95 for $A$. awamori and $A$. oryzae respectively, indicating a strong positive correlation between the colour produced and weight of fungi.

The results from this study revealed that the effect of different carbon sources on sporulation and cell production during the cultivation of $A$. awamori and $A$. oryzae on solid media is substrate dependent. On the other hand, the weight of fungi and colour concentration is constant whatever the age of cultivation and culture conditions may be. Weight of fungi increased with different solid media, which may be related to increasing $\mathrm{C} / \mathrm{N}$ ratio in the solid media (not determined in this case) up to certain levels, depending on the strain, where a drastic reduction in sporulation occurred. From the correlation between the colour produced and the weight of fungi, it was observed that the preferred medium for growth may necessarily be suitable to enhance spore production. It is very obvious that a specific medium formulation is required for the enhancement of growth and sporulation of the specific $A$. awamori and $A$. oryzae strains and that the weight of fungi in these cases correlated strongly with colour production.

\section{Correlation between spores concentration and colour density}

In view of the considerations mentioned above, attention was focused on the development of a standard curve showing a strong relationship between the concentration of spores and colour density. Figure 3 shows the correlation between the concentration of spores harvested at different fermentation times from 24 to $168 \mathrm{~h}$ with colour density measured at wavelength $300 \mathrm{~nm}$. Figure 3 shows correlation coefficients for both fungi growing on different media. The correlation coefficient was higher than 0.96 and 0.98 for $A$. awamori and $A$. oryzae respectively. Nevertheless, comparison of the four regression equations indicated that the amount of colour produced per $10^{6}$ spores/g substrate was different for the four different solid media. The origin of the straight lines was the same but the slopes were different. From this finding, two questions needed to be answered:

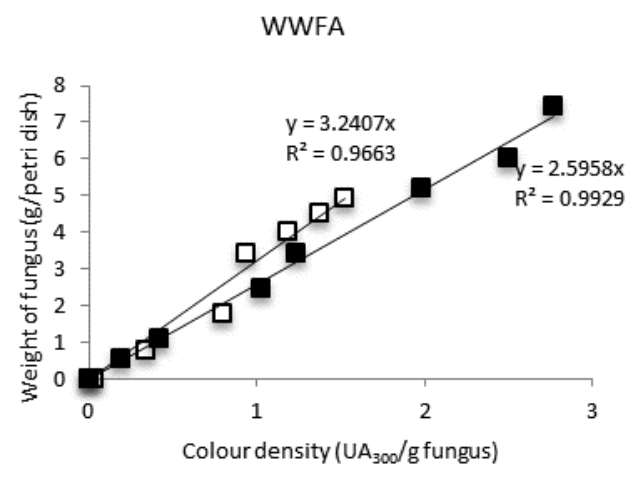

WBA

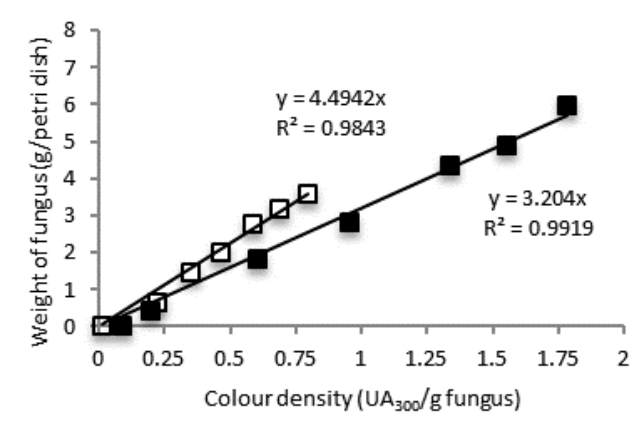

SBHA

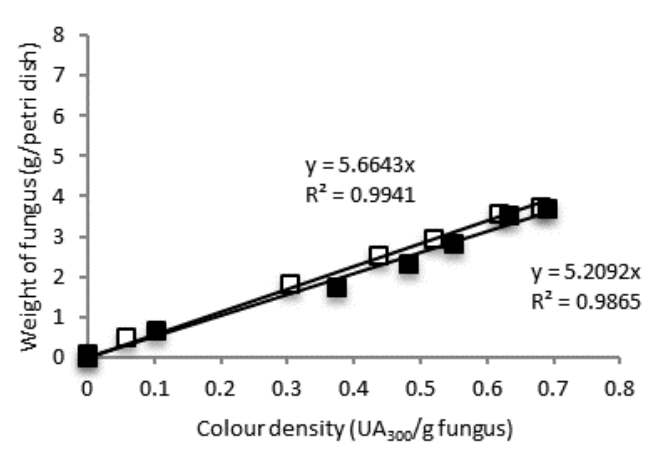

RSMA

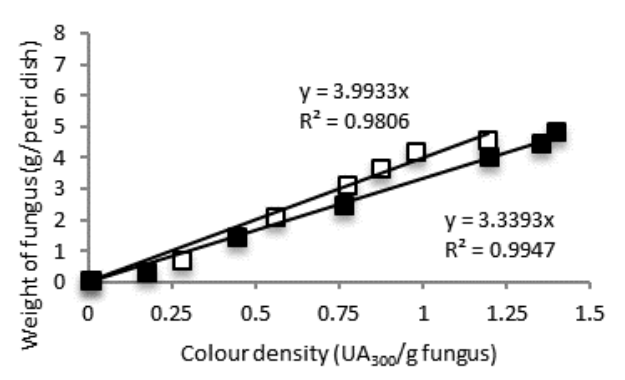

Figure 2: Correlation between weight of fungi and colour of both fungi growing on different solid agar media. [ $\mathbf{\square}_{\text {] }}$ A. awamori; [ $\square$ ]: A. oryzae. [WWFA]: whole wheat flour agar; [WBA]: wheat bran agar; [SBHA]: soybean hulls agar and [RSMA]: rapeseed meal agar. 
(i) is it possible to construct a calibration curve based on the concentration of spores during fungal SSF? (ii) is it possible to use the calibration curve to estimate spores production in fungal SSF?

The linear relationship between spores concentration and absorbance at $300 \mathrm{~nm}$ observed for both fungi on the solid substrates tested was strong and positive where colour density increased proportionally with spores concentration. This result showed it is possible to develop a standard curve based on spore's concentration. Therefore, it would be possible to predict spores production from SSF using colour extracted from fermented mass. Moreover, these results show that spores concentration can be different depending on the fungal strain and the solid substrate.

Different fungi growing on different substrates will produce different colour density. According to Calvo et al. (2002) the ability of fungi to sporulate is related to gene regulation processes, which are affected by environmental and nutritional factors. Temporal and spatial regulation of gene expression, cell specialisation, and intercellular communication may affect the sporulation in the fungal system (Adams et al., 1998). In other words, the correlation between colour density and spores concentration is very satisfactory; however, it does not permit comparison between processes.

\section{Correlation between organic matter loss and colour density}

Dry matter weight of fermented substrate gradually decreased as fermentation progressed, and this was reflected by an increasing percentage of organic matter loss. Organic matter loss is presumably due to loss of $\mathrm{CO}_{2}$ (Kumar et al., 2008). This observation can be related to colour production indicating the gradual utilisation of nutrients in the substrate for biomass and products synthesis. A correlation between organic matter loss and colour density obtained is presented in Figure 4. Colour density increased proportionally with organic matter loss for both fungi. For all substrates tested, the correlation coefficients were greater than 0.98 and 0.96 for $A$. awamori and $A$. oryzae, respectively.

During the fermentation process, the fungus consumes nutrients and it starts producing spores and mycelium. According to Rahardjo et al. (2006) mainly $70 \%$ oxygen is taken up by the aerial hypahae of fungus in SSF. The type of substrate definitely influences the performance of the fungus. Organic matter loss and colour production appear to depend on the medium composition of solid substrate. The figure also shows that both fungi seem to produce the same degree of colour concentration regardless of the solid substrate used. The complexity of fungi in terms of chemical composition, morphology and internal structure suggests that development and colour changes can be indicators that growth happens. The changes in the fungal population can be grouped in changes in the number of cells/spores, morphology, chemical composition (for example colour in this case) and changes in the internal structure (which can be observed under a microscope).

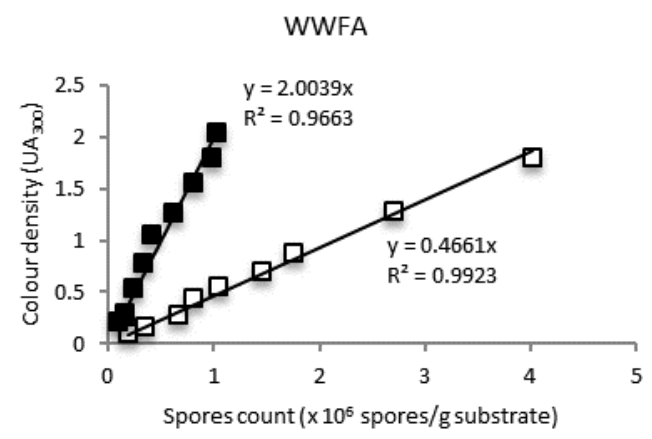

WBA

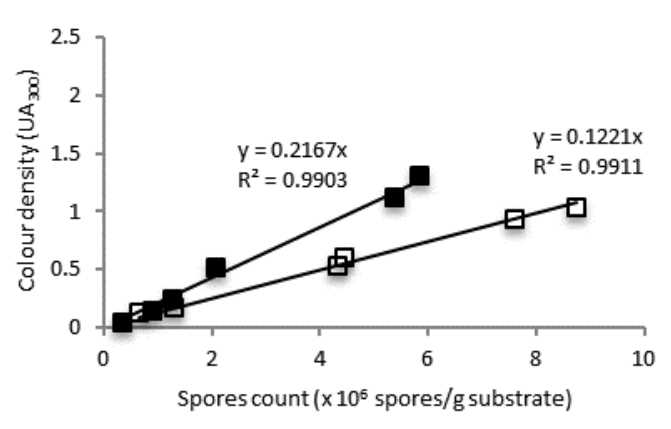

SBHA

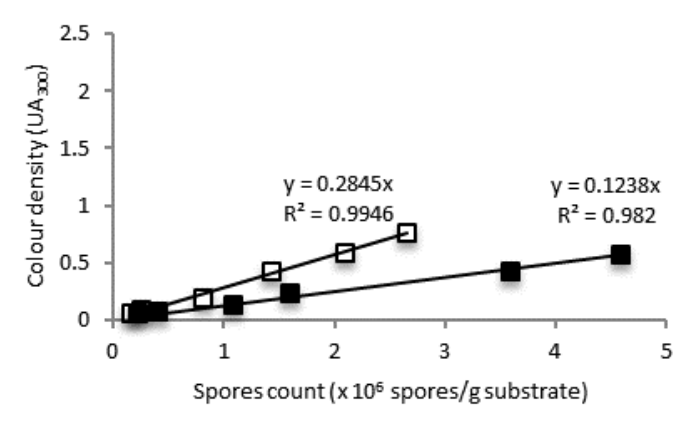

RSMA

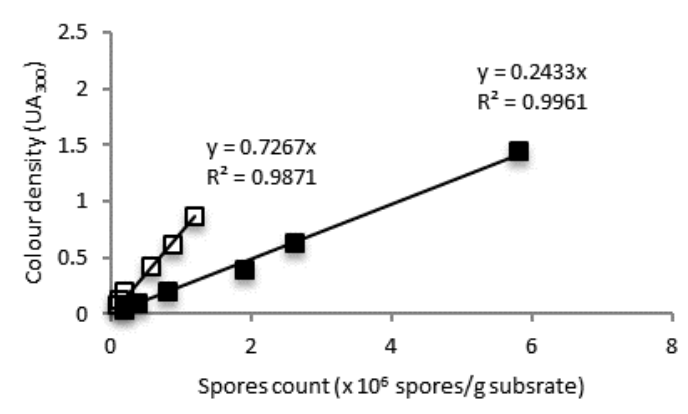

Figure 3: Correlation between spore concentration and colour of

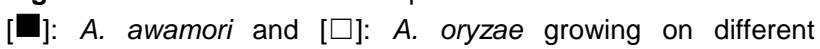
substrate solid agars. [WFA]: whole wheat flour agar; [WBA]: wheat bran agar; [SBHA]: soybean hulls agar and [RSMA]: rapeseed meal agar. 


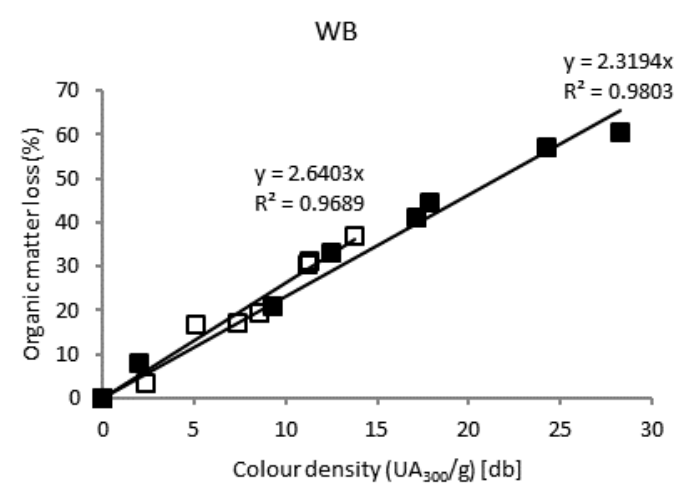

SBH

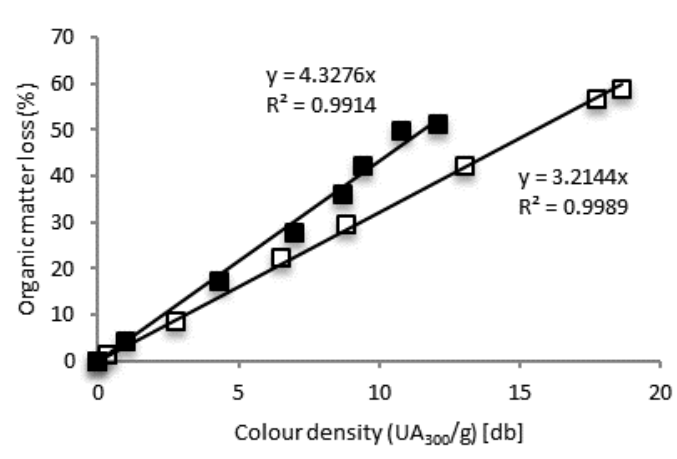

RSM

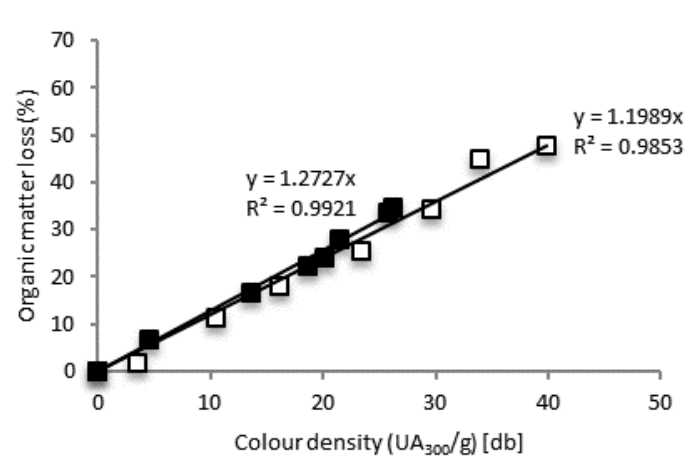

Figure 4: Correlation between organic matter loss and colour density at wavelength $300 \mathrm{~nm}$. [ $\mathbf{D}_{\text {] }}$ A. awamori and $[\square]$ : $A$. oryzae. [WB]: wheat bran; [SBH]: soybean hulls and [RSM]: rapeseed meal

A good correlation between the loss of dry matter weight and $\mathrm{CO}_{2}$ evolution for Trichoderma reesei and Aspergillus candidus growing on different SSF systems (Larroche et al., 1998; Smits et al., 1996, 1998, 1999). Terebiznik and Pilosof (1999) found a good correlation between the loss of dry matter weight and $\mathrm{CO}_{2}$ evolution for $A$. oryzae growing on wheat bran.However, according to Terebiznik and Pilosof (1999), even though this technique is able to estimate biomass in SSF, this is particularly true in the case of fermentation performed without forced aeration.
From a theoretical point of view, it is demonstrated that colour changes as a function of time in fungal SSF can be explained by changes in chemical composition either in the solid material or the fungus itself. Organic matter loss represents one of the indirect techniques used to describe growth of fungi and here correlation clearly indicates that when organic matter loss increases, so does colour production. Viccini et al. (2003) developed a mathematical model for converting the two measurement bases commonly used in the construction of growth profiles in SSF, namely absolute mass ratio (dry biomass/initial dry matter) and relative mass ratio (dry biomass/dry matter). However, they argued that these are not equivalent, due to the loss of dry matter as $\mathrm{CO}_{2}$ during the SSF.

\section{Correlation between glucosamine concentration and colour density}

Glucosamine concentration and colour density in the present study showed a varying pattern between solid substrates tested with regards to time. A. awamori degraded wheat bran at a faster rate than $A$. oryzae. However, A. oryzae degraded rapeseed meal at a faster rate than $A$. awamori. The rate of degradation can be measured by glucosamine concentration and colour density. In this work, a linear correlation was found between the colour produced and glucosamine concentration in fermented material of $A$. awamori and $A$. oryzae (Figure 5). A positive effect of initial moisture content and inoculum size was seen on the concentration of glucosamine and also on the concentration of colour produced. A good correlation was found between colour density and glucosamine concentration using the combined data. The correlation coefficients obtained for wheat bran, soybean hulls and rapeseed meal were higher than 0.97, for both fungi tested. However, comparison of the twelve regression equations indicated that the amount of glucosamine produced per unit absorbance was different for the three solid substrates. Indeed, the origin of the straight lines was the same and the slopes were different for the solid substrates. This shows that the correlation obtained for the effects of inoculum sizes and initial moisture contents was similar for both fungi.

This result provides a useful tool for investigating the use of the correlation of glucosamine concentration with colour changes in the growing fungi for biomass estimation. In essence, high fungal biomass can be reflected by high colour density and high glucosamine concentration. Each of the two fungi produced a different colour density but almost the same concentration of glucosamine. In addition, the same fungus produced different levels of colour density and glucosamine when grown on different substrates.

Referring to the complexity of pigment mixtures produced by Monascus species, it is reported in most studies that pigment composition is determined spectrophotometrically using absorption maxima exhibited around $400 \mathrm{~nm}, 470 \mathrm{~nm}$ and $500 \mathrm{~nm}$ corresponding to yellow, orange and red compounds respectively (Juzlova et al., 1996; Lin et al., 1992). In the case of Monascus species, intensive research has been carried out to identify the compounds responsible for the colour. The 
pigment produced by this microorganism has been used successfully to describe growth in either SmF or SSF.

Results presented in this section show that the colourimetric technique could be considered as a reliable indirect method for estimating fungal biomass in the case of both fungal species. Figure 5 shows that glucosamine concentration appears to depend on the solid substrate used. In other words, glucosamine concentration depends on the medium composition of the solid substrate. Indeed, the level of glucosamine was affected by the nature of the solid substrate (carbon and nitrogen source), but not by the type of microorganism used or the treatment
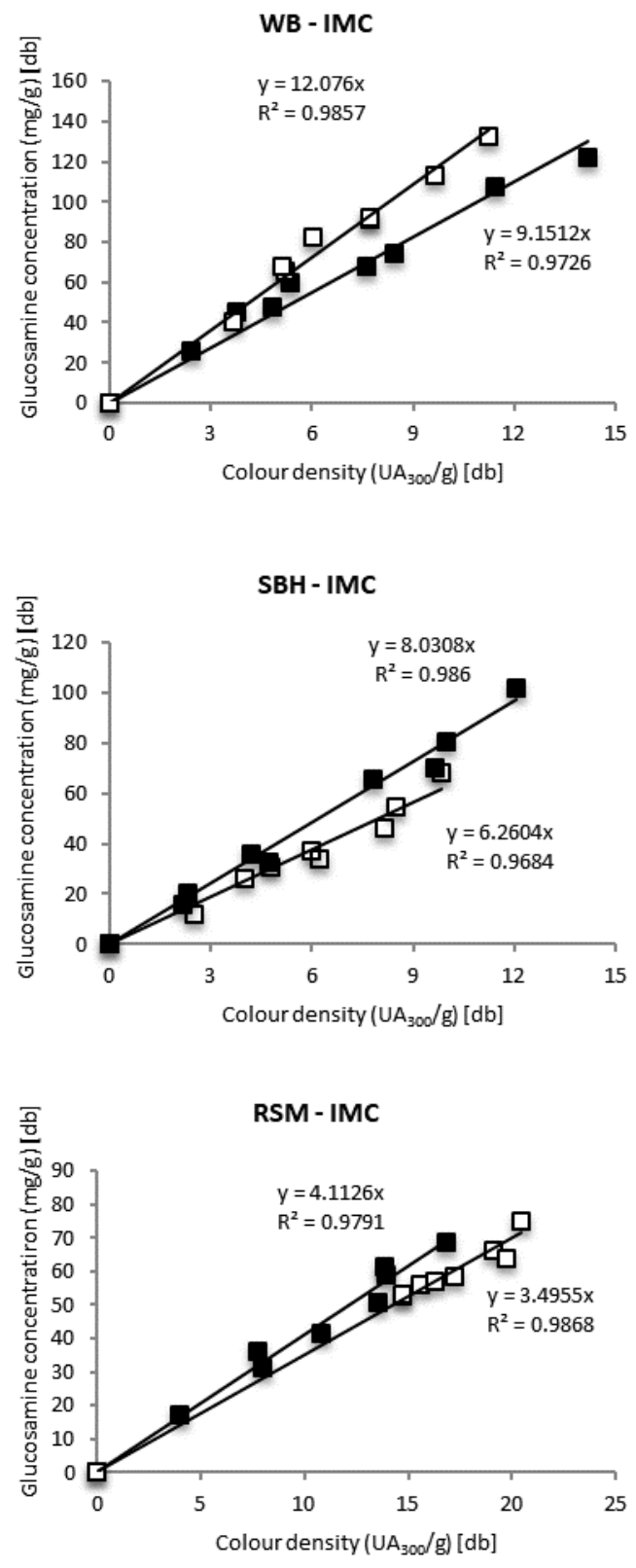

conducted (the effect of inoculum size and initial moisture content in this study).

This study also demonstrates the strong and positive correlation between colour density and glucosamine concentration. Therefore, it is possible to make a good estimation of the level of glucosamine using the colourimetric method developed in this study. By using the linear regression equation obtained in SSF for each fungus and solid substrate, quantities of colour measured could be easily converted into milligram glucosamine per gram of dry solid substrate (on a dry basis) (mg/g [db]).
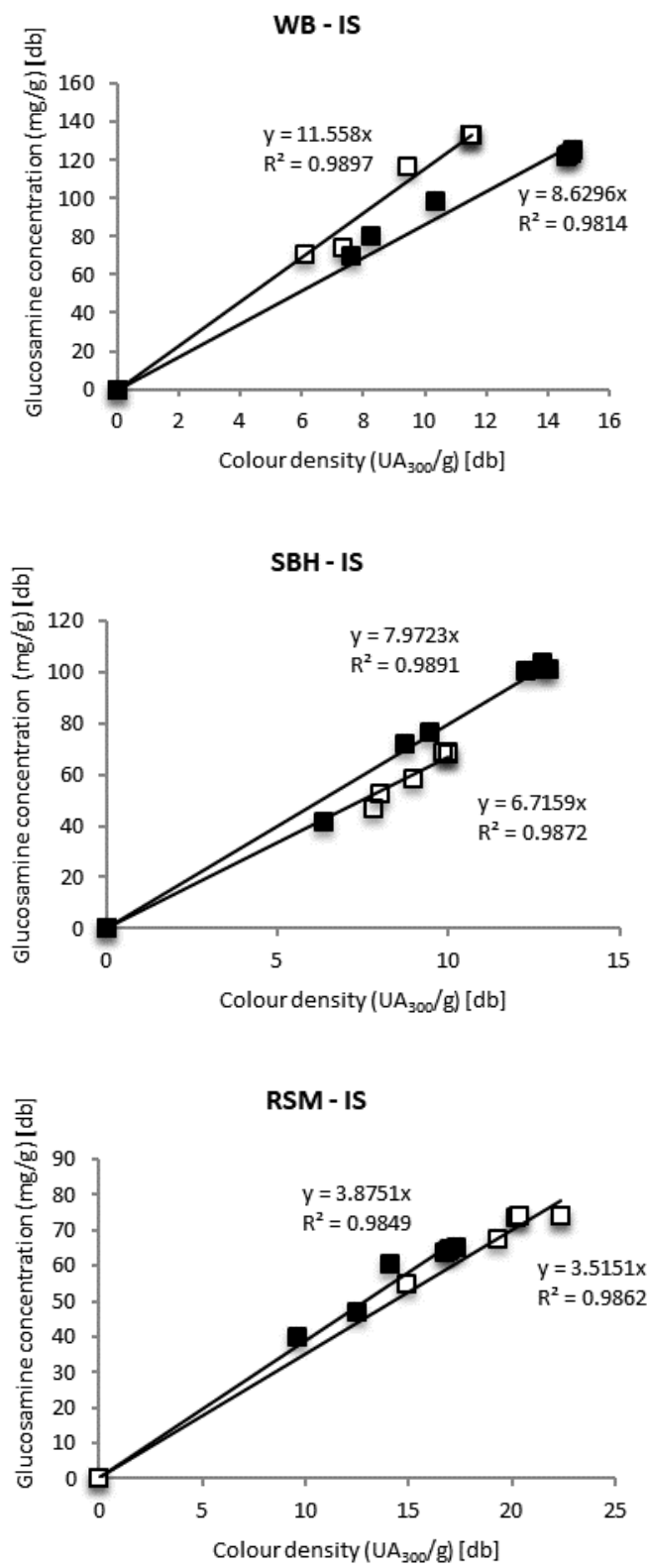

Figure 5: Correlation between colour produced and concentration of glucosamine tested at different initial moisture content [IMC] and inoculum size [IS] for both [ ${ }^{\text {] }}$ : A. awamori and [ $\square$ ]: $A$. oryzae on different substrates. [WB]: wheat bran; [SBH]: soybean hulls and [RSM]: rapeseed meal. 


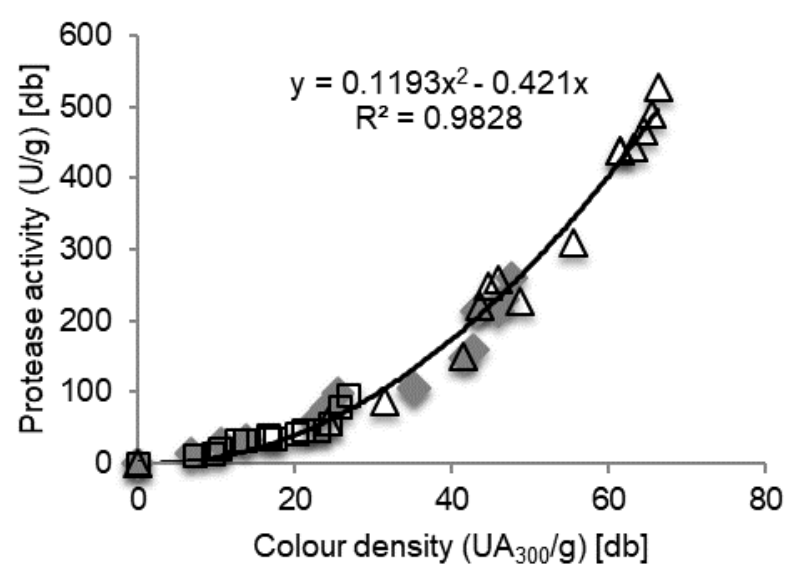

Figure 6: Correlation between colour produced and protease activity at different initial moisture contents and inoculum sizes for three different substrates. ( $)$ ): wheat bran; $(\square)$ : soybean hulls and $(\Delta)$ : rapeseed meal.

\section{Correlation between enzyme activities and colour density}

In this study, the colour extract was also used as a supernatant crude enzyme extract for further analysis by determining the activity of enzymes such as glucoamylase, protease, xylanase and cellulase in order to investigate whether a correlation exists between the activity of enzymes within the extract and the intensity of its colour. It is worth noting from visual observation that different colour density of supernatant crude enzyme extract will give different activity of enzymes. The darker will results high enzymes activity. This phenomenon also observed by Ariff (1993) that high production of glucoamylase was accompanied by high production of yellow pigments. Therefore, in the SSF experiments used in this work, the activity of protease is used as a reference to verify how efficient the applied technique to quantify the biomass growth during the process was.

Figure 6 shows correlation between protease activities and colour density extracted from fermented $A$. awamori and $A$. oryzae on wheat bran, soybean hulls and rapeseed meal. Experiments were carried out at different initial moisture contents and inoculum sizes. Cell free extracts were obtained from fermented fungal biomass after $72 \mathrm{~h}$ of fermentation. The results showed a positive and significant correlation between colours produced by fungal SSF and protease activity. In contrary with results presented above, it was observed that colour density exponentially increases with the protease activity. The production of enzymes and pigments would be independent on solid substrates and fungi.

Aspergillus awamori and $A$. oryzae showed a satisfactory growth and produced high protease activity and high colour concentration on rapeseed meal followed by wheat bran and soybean hulls. This is expected since the composition of rapeseed meal is known to be rich in protein content (38.9\%) (Wang et al., 2010). The results obtained in this work for enzyme production were similar to those reported by Ramana Murthy et al., (1993), who estimated $A$. niger growth in wheat bran using reflected light. It should borne in mind that the colour produced cannot be attributed a single substance; it might involve one or more substances and therefore it cannot be attributed to a single wavelength. Dutra et al. (2008) and Couri et al. (2006) reported that the digital image process technique could be used to monitor biomass growth in a SSF process. They observed that the image processing have a linear relationship with lipase activity (Dutra et al., 2008) and glucosamine (Couri et al., 2006), and to correlate biomass growth.

\section{CONCLUSION}

The results of this study indicated that the colourimetric technique could be used to monitor biomass growth in a SSF process and to correlate biomass growth and weight fungus, spores concentration, organic matter loss, glucosamine and enzyme activity. Overall, the estimation of colour density through UV-Vis spectrophotometry from SSF would be a favourable indirect method to describe the growth of fungi. A higher number of experiments and improvements on the colour changes technique in SSF using various microorganisms and solid substrates are needed to validate the technique. Further studies should be carried out to obtain more data to support the colourimetric technique using different fungi and bacteria, and also using different types of solid substrate whether in single form or mixed-solid substrate from different solid wastes. In addition to SSF, the technique based on colour changes for biomass estimation should also be extensively studied in SmF using filamentous fungi with solid substrate particles. No method is ideally suited to all situations and hence the most appropriate method to a particular fermentation application must be chosen in each case based on the simplicity of the procedure, its cost and its accuracy.

\section{ACKNOWLEDGEMENT}

To the Malaysian Agricultural Research and Development Institute (MARDI) and Government of Malaysia for providing me an opportunity to further study and financial support during my study.

\section{REFERENCES}

Abdul Manan, M. and Webb, C. (2018a). Estimation of growth in solid state fermentation: A review. Malaysian Journal of Microbiology 14(1), 61-69.

Abdul Manan, M. and Webb, C. (2018b). Estimating fungal growth in submerged fermentation in the presence of solid particles based on colour development. Biotechnology and Biotechnological Equipment 32(3), 618-618.

Abdul Manan, M. and Webb, C. (2016). Extracted substrate colour as an indicator of fungal growth in solid state fermentation. Malaysian Journal of Microbiology 12(6), 445-449. 
Adams, T. H., Wieser, J. K. and Yu, J. -H. (1998). Asexsual sporulation in Aspergillus nidulans. Microbiology and Molecular Biology Reviews 62(1), 35-54.

Aidoo, K. E., Hendry, R. and Wood, B. J. B. (1982). Solid substrate fermentations. Advances in Applied Microbiology 28, 201-237.

Ariff, A. (1993). The influence of mode of operation on the production of glucoamylase by Aspergillus awamori. $\mathrm{PhD}$ Thesis. University of Manchester Institute of Science and Technology, United Kingdom.

Borzani, W. (2000). A weighing method to identify the microbial growth phases in solid-state fermentation tests. World Journal of Microbiology and Biotechnology 16(7), 601-605.

Calvo, A. M., Wilson, R. A., Bok, J. W. and Keller, N. P. (2002). Relationship between secondary metabolism and fungal development. Microbiology and Molecular Biology Reviews 66(3), 447-459.

Chattaway, T., Demain, A. L. and Stephanopoulos, G. (1992). Use of various measurements for biomass estimation. Biotechnology Progress 8(1), 81-84.

Couri, S., Merces, E. P., Neves, B. C. V. and Senna, L. F. (2006). Digital image processing as a tool to monitor biomass growth in Aspergillus niger 3T5B8 solid-state fermentation: preliminary results. Journal of Microscopy 224(3), 290-297.

Dutra, J. C. V., Terzi, S. C., Bevialaqua, J. V., Damaso, M. C. T., Couri, S., Langone, M. A. P. and Senna, L. F. (2008). Lipase production in solid-state fermentation monitoring biomass growth of Aspergillus niger using digital image processing. Applied Biochemical and Biotechnology 147(1-3), 63-75.

Harris, C. M. and Kell, D. B. (1985). The estimation of microbial biomass. Biosensors 1, 17-84.

Hashemi, M., Mousavi, S. M., Razavi, S. H. and Shojaosadati, S. A. (2011). Mathematical modelling of biomass and $\alpha$-amylase production kinetics by Bacillus sp. in solid-state fermentation based on dry weight variation. Biochemical Engineering Journal 53, 159-164.

John, M. R. and Stuart, D. M (1991). Production of pigments by Monascus purpureus in solid culture. Journal of Industrial Microbiology 8(1), 23-28.

Juzlova, P., Martinkova, L. and Kren, V. (1996). Secondary metabolites of the fungus Monascus: A review. Journal of Industrial Microbiology 16(3), 163170.

Juzlova, P., Martinkova, L., Lozinski, J. and Machek, F., (1994). Ethanol as substrate for pigment production by the fungus Monascus purpureus. Enzyme and Microbial Technology 16(11), 996-1001.

Kabanova, N., Stulova, I. and Vilu, R. (2012). Microcalorimetric study of the growth of bacterial colonies of Lactococcus lactis IL1403 in agar gels. Food Microbiology 29, 67-79.

Koutinas, A. A., Wang, R. and Webb, C. (2003). Estimation of fungal growth in complex, heterogenous culture. Biochemical Engineering Journal 14, 93-100.
Kumar, A. and Jain, V. K. (2008). Solid state fermentation studies of citric acid production. African Journal of Biotechnology 7(5), 644-650.

Larroche, C., Moskia, J. and Gros, J. B. (1998). A convenient method for initial dry weight determination in samples from solid state cultivations. Process Biochemistry 33, 447-451.

Lie, S. (1973). EBC [European Brewery Convention]ninhydrin method for determination of free $\alpha$-amino nitrogen. Journal of the Institute of Brewing 79, 37-41.

Lin, T. F., Yakushijin, K., Buchi, G. H. and Demain, A. L., (1992). Formation of water-soluble Monascus red pigments by biological and semi-synthetic processes. Journal of Industrial Microbiology 9(3), 173-179.

Mitchell, D. A., de Lima Luz, L. F. and Krieger, N. (2011). Bioreactors for solid-state fermentation. Reference Module in Life Sciences - Comprehensive Biotechnology 2, 347-360.

Mitchell, D. A., Krieger, N. and Berovic, M. (2010). Solid-state fermentation bioreactor fundamentals Introduction and overview. In: Solid state fermentation bioreactors - Fundamentals of design and operation. Mitchell, D. A., Krieger, N. and Berovic, M. (eds.) Springer-Verlag Berlin Heidelberg, Germany. pp. 1-12.

Mitchell, D. A., Berovic, M., Nopharatana, M. and Krieger, N. (2006). Introduction to solid-state fermentation bioreactors. In: Solid-state fermentation bioreactors: Fundamentals of design and operation. Mitchell, D. A., Krieger, N. and Berovic, M. (eds.) Springer-Verlag Berlin Heidelberg. pp. 33-43.

Mitchell, D. A., von Meien, O. F., Krieger, N. and Dalsenter, F. D. H. (2004). A review of recent developments in modelling of microbial growth kinetics and intraparticle phenomena in solid-state fermentation. Biochemical Engineering Journal 17, 1526.

Rahardjo, Y. S. P., Tramper, J. and Rinzema, A. (2006). Modeling conversion and transport phenomena in solid-state fermentation: A review and perspectives. Biotechnology Advance 24, 161-179.

Ramana Murthy, M. V., Thakur, M. S. and Karanth, N. G. (1993). Monitoring of biomass in solid state fermentation using light reflectance. Biosensors and Bioelectronics 8, 59-63.

Scotti, C. T., Vergoignan, C., Feron, G. and Durand, A. (2001). Glucosamine measurement as indirect method for biomass estimation of Cunninghamella elegans grown in solid state cultivation conditions. Biochemical Engineering Journal 7(1), 1-5.

Smits, J. P., van Sonsbeek, H. M., Tramper, J., Knol, W., Geelhoed, W., Peeters, M. and Rinzema, A. (1999.) Modelling fungal solid-state fermentation: The role of inactivation kinetics. Bioprocess Engineering 20, 391-404.

Smits, J. P., Rinzema, A., Tramper, J., Van Sonsbeek, H. M., Hage, J. C., Kaynak, A. and Knol, W. (1998). The influence of temperature on kinetics in solid-state fermentation. Enzyme and Microbial Technology 22, 50-57.

Smits, J. P., Rinzema, A., Tramper, J., Van Sonsbeek, H. M. and Knol, W. (1996). Solid-state fermentation of 
wheat bran by Trichoderma reesei QM9414: Substrate composition changes, C balance, enzyme production, rowth and kinetics. Applied Microbiology and Biotechnology 46, 489-496.

Swift, M. J. (1973). The estimation of mycelial biomass by determination of the hexosamine content of wood tissue decayed by fungi. Soil Biol Biochemistry 5(3), 321-332.

Terebiznik, M. R., and Pilosof, A. M. R. (1999). Biomass estimation in solid state fermentation by modeling dry matter weight loss. Biotechnology Technique 13, 215219.

Viccini, G., Mitchell, D. A. and Krieger, N. (2003). A model for converting solid state fermentation growth profiles between absolute and relative measurement bases. Inter-Conversion of Growth Profiles in SSF. Food Technology and Biotechnology 41(3), 191-201.

Wang, R. -H, Shaarani, S. M., Godoy, L. C., Melikoglu, M., Vergara, C. S., Koutinas, A. and Webb, C. (2010). Bioconversion of rapeseed meal for the production of a generic microbial feedstock. Enzyme and Microbial Technology 47(3), 77-83.

Wang, R. -H (1999). Continuous production of generic fermentation feedstock from whole wheat flour. PhD Thesis. The University of Manchester Institute of Science and Technology, Manchester, United Kingdom.

Wei, P. L., Cen, P. L. and Sheng, C. Q. (2006). Comparison of three biomass estimation methods in solid state fermentation. Journal of Food Science and Biotechnology 25(1), 60-64.

Wong, H. C. and Koehler, P. E. (1981). Production and isolation of an antibiotic from Monascus purpureus and its relationship to pigment production. Journal of Food Science 46(2), 589-592. 